\title{
Essais
}

Revue interdisciplinaire d'Humanités

Hors-série 7 | 2022

Récits d'outre-thèse

\section{Le récit d'un Sahélien devenu docteur}

Hassane Mahamat Hemchi

\section{(2) OpenEdition}

Journals

Édition électronique

URL : https://journals.openedition.org/essais/10848

DOI : 10.4000/essais. 10848

ISSN : 2276-0970

\section{Éditeur}

École doctorale Montaigne Humanités

\section{Édition imprimée}

Date de publication : 15 avril 2022

ISBN : 979-10-970024-00-0

ISSN : 2417-4211

\section{Référence électronique}

Hassane Mahamat Hemchi, «Le récit d'un Sahélien devenu docteur », Essais [En ligne], Hors-série 7 |

2022, mis en ligne le 01 février 2022, consulté le 17 janvier 2023. URL : http://

journals.openedition.org/essais/10848; DOI : https://doi.org/10.4000/essais.10848

Ce document a été généré automatiquement le 17 janvier 2023.

Tous droits réservés 


\title{
Le récit d'un Sahélien devenu docteur
}

\author{
Hassane Mahamat Hemchi
}

Il n'y a ni livre, ni auteur, ni expert qui puisse résoudre vos problèmes, il n'y a que vous-même.

Ce sont vos problèmes à vous et c'est à vous de vous débrouiller ${ }^{1}$.

1 L'objectif d'une thèse de doctorat, c'est de pouvoir mener de façon autonome et experte un projet de recherche scientifique qui apporte ou qui doit apporter une contribution originale à la connaissance, à l'interprétation ou au développement scientifique et/ou technologique ${ }^{2}$. Mais pour atteindre cet objectif dans l'écriture d'une thèse en sciences sociales beaucoup de facteurs s'entremêlent, entre contingences et nécessités ${ }^{3}$. Ce qui montre à suffisance que l'on doit être capable d'avoir une certaine posture épistémologique, et surtout une certaine compétence de base que l'on doit développer avec l'évolution du projet de thèse. Bien que certains pensent que tout a été dit, même si "rien ne se perd, rien ne se crée, tout se transforme ${ }^{4}$ ", alors il s'agit de dire autrement. Ce qui fait et/ou qui fera la particularité, à travers la manière d'écrire, de contextualiser, les angles d'attaque du problème choisis, les outils utilisés, les méthodes arpentées, l'ouverture d'esprit - tout cela démontrera amplement la capacité d'adaptation, d'avancement et d'aboutissement d'une thèse de doctorat.

\section{Présentation et processus d'évolution professionnelle}

2 Hassane MAHAMAT HEMCHI, de nationalité tchadienne ${ }^{5}$, né d'une mère Téda (Tibesti) et d'un père Dozi (Borkou). Un Sahélien, d'une mère qui n'est jamais allée à l'école, et un père infirmier diplômé d'État et puis réorienté vers l'ophtalmologie (lauréat de l'institut d'ophtalmologie tropicale d'Afrique) de Bamako au Mali. J'ai eu une enfance atypique ; j'étais d'abord derrière les chèvres, puis dans les champs comme agriculteur par le biais de la pratique de chadouf ${ }^{6}$. Et enfin, j'étais aussi à l'école coranique. Rien ne 
prédisait que j'aurais un jour mon doctorat, et que je deviendrais enseignant-chercheur dans une institution panafricaine.

3 Je suis actuellement enseignant-chercheur permanent au sein de l'École Africaine des Métiers de l'Architecture et de l'Urbanisme (EAMAU) de Lomé (Togo). Une école interÉtats créée en 1975 par les chefs d'États africains et qui regroupe 14 pays membres. Urbaniste de formation et spécialiste des mobilités urbaines, je suis détenteur d'un Diplôme Universitaire de Technologie de l'École Supérieure de Technologie de Salé (Maroc), d'une Maîtrise ès Science et Technique de la Faculté des Sciences et Techniques de Mohammedia (Maroc), d'un Diplôme des Études Supérieures en Aménagement et Urbanisme de l'Institut National d'Aménagement et d'Urbanisme de Rabat (Maroc). Je suis également titulaire d'un Master en Paysage et Évaluation Environnementale dans les Projets d'Urbanisme et de Territoires de l'Institut d'Aménagement, de Tourisme et d'Urbanisme de l'ex-université de Bordeaux III et enfin, d'un Doctorat en Aménagement de l'Espace et Urbanisme de l'Université Bordeaux Montaigne (France).

4 J'ai été enseignant-chercheur à l'École Nationale Supérieure des Travaux Publics de N'Djamena (Tchad) d'octobre 2008 à janvier 2019 (Chef de Département de Topographie et Cartographie de ladite école entre septembre 2016 à janvier 2019). Enfin, j'ai collaboré auprès de divers acteurs, en tant que consultant indépendant, à des études et projets opérationnels que j'ai parfois pilotés aussi.

5 Mes travaux de recherche portent aujourd'hui sur les études urbaines dans le contexte de villes africaines ; d'abord, en matière de planification urbaine, de gestion urbaine, de mobilité urbaine et de la protection de l'environnement. Bien que mon parcours semble riche à certains, j'ai surtout voulu faire une thèse de doctorat, depuis mon jeune âge. Le choix du thème de mes travaux de doctorat s'explique par le fait que je suis un urbaniste de formation et que j'ai constaté que la ville de N'Djamena vit au quotidien une discrimination spatiale, due à la disposition des populations qui se déplacent avec des modes de transports collectifs de type artisanal.

6 Lors de mes études supérieures entre universités et instituts du Royaume Chérifien ${ }^{7}, \mathrm{j}^{\prime}$ ai eu l'occasion de travailler entre la maîtrise et le diplôme d'études approfondies auprès des bureaux d'études techniques d'architecture et d'urbanisme. Après sept années d'études, je suis retourné au Tchad pour intégrer le Projet d'Appui au Développement Urbain. C'est un projet public qui a été soumis au financement de la Banque Mondiale. En effet, le projet a été initié par le Ministère de l'Aménagement du Territoire, de l'Urbanisme et de l'Habitat dans le cadre de l'appui à la Stratégie Nationale de Réduction de la Pauvreté au Tchad. Le projet concerne cinq villes du pays (N'Djamena, Moundou, Sarh, Abéché et Doba). J'avais soumis ma candidature en ligne depuis le Maroc et reçu un avis favorable pour le recrutement. Mais une fois sur place, il a d'abord été question d'être évalué par les directeurs techniques du Ministère. Ensuite, il se trouve que je suis trop jeune à leurs yeux. J'ai dû passer un test pour vérifier l'authenticité des diplômes et du niveau de formation, recommandé par le Secrétaire Général de l'époque, exercice ayant une connotation discriminatoire implicite. Mes soucis ne se limitent toutefois pas à mon jeune âge. Mais du fait que; Pour le Secrétaire Général du ministère, je dois renoncer au poste sur lequel je viens d'être recruté. Si je veux à tout prix rejoindre le projet, je n'aurai que le salaire d'un agent du ministère dans la fonction publique ${ }^{8}$, chose qui est en contradiction avec la fiche de poste. Je suis obligé de décliner. 
J'ai préféré par la suite aller enseigner à l'ENSTP qui relève du Ministère des Infrastructures, des Transports et de l'Aviation Civile de l'époque. C'est ainsi que je suis devenu enseignant permanent de l'École Nationale Supérieure des Travaux Publics.

Il faut signaler que je m'étais inscrit en thèse au Maroc avant de revenir au Tchad. C'est pourquoi je voulais rejoindre le milieu de l'enseignement et de la recherche. J'ai pris service à ladite école en octobre 2008. Puis, en février 2009, je suis reparti au Maroc pour renouveler mon inscription en thèse. Lors de ce séjour, on m'a demandé de suivre des cours sur place et d'assumer aussi le rôle d'Assistant Technique d'Enseignement et de la Recherche. De nouvelles exigences instituées par la nouvelle réglementation des écoles doctorales au Maroc. Face à cette situation, je me suis vu dans l'obligation d'abandonner la thèse.

En juin 2010, j'ai envoyé une demande d'admission en master à l'Institut d'Aménagement, de Tourisme et de l'Urbanisme. Le gouvernement français octroyait à ce moment-là des bourses de mérite dans le cadre de la formation des élites africaines à travers les institutions d'enseignement supérieur des pays en développement. C'est dans ce cadre que ma candidature a été retenue pour une bourse du gouvernement français via l'ambassade de France au Tchad pour le compte de l'ENSTP. Mon choix s'est porté sur Bordeaux qui offrait des parcours master en urbanisme. C'est en octobre 2010 que je suis arrivé en France. Il faut mentionner que la réponse d'acceptation de l'université précisait que la formation durerait 10 mois ; mais sur place, il s'est avéré que la formation s'étalerait sur deux ans. Une première année avec des cours magistraux et des examens. Et une deuxième année qui ferait l'objet d'un stage obligatoire de six mois afin que le rapport élaboré soit défendu publiquement devant un jury. Ces précisions m'ont incité à faire une demande de prolongation de bourse, qui a été acceptée mais sans indication sur l'échéance de prise en charge. J'ai validé la première année (2010-2011) puis, avant de partir en vacances, j'ai déposé une demande de candidature au cycle de doctorat. À la rentrée en septembre 2011, j'ai eu une réponse favorable à ma demande sur la base de l'équivalence du diplôme étranger.

Il faut avouer que la décision la plus dure à prendre vient à point nommé ; c'est-à-dire : faire un choix entre rester professionnel ou entamer la carrière d'enseignantchercheur, à commencer par la thèse. J'ai toujours voulu faire un doctorat, et enfin je suis accepté dans une université occidentale, mais sans financement. Il me revient de choisir la thèse, tout en sachant que je vais devoir m'autofinancer.

Le sujet de mes travaux de doctorat était «les mobilités urbaines et planification. Cas de la ville de N'Djamena $»^{9}$. L'objectif était de faire l'étude de la mobilité urbaine et des caractéristiques de déplacements des habitants de N'Djamena, en mettant en relation leurs pratiques avec les composantes économiques et sociales au sein d'une ville en pleine mutation. À ce stade, je n'avais pas encore de master, qui devait être validé par un stage de six mois. Alors j'ai décidé d'aller au Maroc pour un séjour de six mois afin de remplir cette condition. C'est ainsi qu'une fois effectuée l'inscription administrative au cycle du doctorat, je suis parti en stage en bureau d'étude technique d'architecture et d'urbanisme de janvier à fin juin 2012, tout en travaillant sur la thèse. De retour en juillet à Bordeaux, il était déjà trop tard pour que je puisse soutenir. Ce sera fait à la rentrée de septembre 2012. 


\section{La période de rédaction de la thèse}

11 Le vrai travail de la thèse proprement dit a commencé lors de ma deuxième année d'inscription. Il faut préciser que mon engagement avec l'ENSTP m'a permis de mettre en place une convention de cotutelle entre l'université de N'Djamena et celle de Bordeaux, même si cette dernière n'a pas donné les résultats escomptés, l'école doctorale de l'université de N'Djamena n'ayant pas été créée. Mais, dans la convention, toutes les réinscriptions étaient programmées à Bordeaux, ce qui m'a permis de faire facilement des va-et-vient entre mon terrain d'étude (N'Djamena) et la France. Comme je l'ai dit plus haut, la bourse de master dont je bénéficiais a pris fin en février 2012, lorsque j'étais en stage au Maroc. Une fois de retour à Bordeaux, je suis obligé d'aller travailler pour payer le loyer bien logé en cité universitaire et, surtout, pour garantir les frais de réinscription au cycle doctoral. Je l'ai fait dans l'agroalimentaire en tant qu'ouvrier, pour ne pas abandonner la thèse.

12 En novembre 2012, je me suis réinscrit, et suis rentré pour mon premier terrain à N'Djamena (Tchad), la capitale tchadienne (N'Djamena); subdivisée en dix arrondissements, elle s'étend sur une superficie de plus de 20000 ha; la population est estimée à plus de 1207500 habitants en 2013, et représente $45 \%$ de la population urbaine du pays. Il était important pour moi de voir de manière précise le phénomène de la mobilité urbaine à travers les trois arrondissements enquêtés : le 1er, le 8e et le 9e. Le travail de terrain consistait à mener des enquête-ménage de mobilité urbaine, j'avais pris un effectif de 333 ménages sur les trois arrondissements d'enquête soit une répartition de 111 ménages par arrondissement. Enfin, j'ai retenu 150 entretiens répartis équitablement entre les trois catégories de moyens de transport en commun (taxis urbains, minibus et mototaxis).

13 Lors de mes séjours à Bordeaux, je rédigeais, exploitais mes résultats et avançais le plus possible dans mes travaux, tout en participant aux différentes activités offertes aux doctorants par l'École Doctorale. La Maison des Suds (MDS) du laboratoire Passages, demeure jusqu'à aujourd'hui l'un des lieux idéaux pour la rencontre des doctorants, chercheurs, enseignants-chercheurs, étudiants, etc. soit à la bibliothèque, soit pendant les différentes activités scientifiques hébergées dans ses locaux. Il faut signaler que le laboratoire Les Afriques dans le Monde est aussi logé en partie dans la même enceinte que le laboratoire Aménagement, Développement, Environnement, Santé, Sociétés (ADESS). Ce dernier était mon laboratoire d'attache. Se rencontraient là différents doctorants ayant pour terrains les Suds d'une manière générale, et l'Afrique en particulier. C'est dans ces locaux que nous avons pu faire les premières réunions pour la création du Réseau Aquitain des Jeunes Africanistes avec nos confrères du laboratoire associé. Il y avait aussi l'Association bordelaise des doctorants et étudiants de masters en sciences de l'espace et du territoire (Doc Géo) qui participait à l'animation de la vie du laboratoire sur le Campus.

Ainsi, dans la vie de l'université en général et du laboratoire en particulier, j'ai dû faire partie aussi des listes recevables pour les élections à la commission de la recherche du conseil académique de l'université au compte des Associations Réunies (ARB3Inter'Assos).

14 Par ailleurs, il faut signaler qu'il y a une panoplie de petites formations ${ }^{10}$ d'apprentissage, de perfectionnement et/ou d'accompagnement tout au long de chaque année académique au profit des futurs docteurs. C'est un écosystème dynamique, 
inclusif et aussi compétitif. Ce qui n'est pas le cas dans certaines villes, d'après les témoignages de camarades doctorants venus d'ailleurs. Enfin, il faut dire que toute cette ambiance exceptionnelle si j'ose dire, est aussi due en grande partie au personnel que l'on croise dans les différents services, à l'École doctorale comme à la Maison des Suds. Des personnes pleines d'âme qui aident, accompagnent chaque année de nouveaux visages vers la réussite de leurs idéaux dans la production cartographique, la préparation de terrains, l'analyse des données, etc. Des professeurs, des assistants administratifs, en passant par des stagiaires, des bibliothécaires et aussi des personnes de passage pour une manifestation scientifique, de quelques heures à quelques jours.

Il faut préciser que tout n'a pas été rose : être doctorant, c'est aussi vivre une période de galère, d'incertitudes que certains n'oseront jamais conseiller à leurs progénitures, parce qu'en plus de ses propres problèmes, l'on doit faire face à des questions existentielles. Je dirais qu'une thèse ne finit jamais. Il faut savoir mettre fin à son travail tout en fixant des objectifs à travers un agenda très exigeant. Les travaux réalisés doivent être valorisés en continu dans sa propre université ou ailleurs. Sinon, on finit par perdre confiance.

16 J'ai soutenu ma thèse de doctorat le 23 juin 2015. Pour moi, c'était la fin de douze années de challenge ${ }^{11}$ et en même temps le début d'une nouvelle vie d'enseignantchercheur à laquelle j'aspire, même si je le suis déjà avec l'École Nationale Supérieure des Travaux Publics de N'Djamena. J'ai soutenu devant un jury composé de six membres qui m'a accordé la mention très honorable. Il faut signaler que la version du document de la soutenance a été publiée en l'état et qu'elle se retrouve aujourd'hui à plus de 30000 téléchargements ${ }^{12}$ sur le site des archives ouvertes. Je suis rentré au Tchad pour enfin rejoindre ma famille deux jours après la soutenance.

Chaque thèse a son histoire et chaque travail de thèse dépend de celui qui le fait, de sa détermination et de son pouvoir d'automotivation. La réussite d'une thèse de doctorat dépend de plusieurs facteurs. Il s'agit d'abord d'aimer ce qu'on fait, d'aimer lire et surtout beaucoup lire, avec passion. Écrire et réécrire plusieurs fois un texte et aussi se faire lire par un tiers avant de soumettre un travail ${ }^{13}$. La réussite d'une thèse est aussi liée à l'encadrant ; ce dernier doit être humain, attentif, disponible et rigoureux dans le travail. Puis le milieu dans lequel on fait la thèse peut jouer un rôle dans la réussite ou l'échec.

Si vous êtes ouvert aux autres, à travers une "thérapie de groupe », vous n'allez pas déprimer; vous allez comprendre que vous n'êtes pas le seul à vous retrouver quelquefois sans repères. Ces rencontres qui vous permettent de vous poser des questions sur la vie courante, sur l'avenir, et parfois vous amènent à vous demander pourquoi avoir voulu faire une thèse remontent le moral, permettent d'avoir confiance en soi et de persévérer à tout prix ${ }^{14}$. La thèse est individuelle certes, mais se concrétise avec l'aide de beaucoup de personnes. La thèse peut vous mener à des réflexions d'abandon, quand vous êtes marié ou que vous avez déjà une situation stable; une vie que la majorité pense avoir après la thèse.

\section{La vie d'après thèse}

Après la soutenance, j'ai eu des opportunités; d'abord en tant qu'enseignantchercheur, puis expert consultant auprès de certaines institutions ${ }^{15}$. Le titre de docteur m'a permis de soumettre ma candidature, en tant qu'urbaniste et spécialiste des 
mobilités urbaines, à des offres de formations et des études opérationnelles. Ces occasions m'ont permis d'avoir une certaine autonomie financière. Enfin, ce titre m'a permis de voyager dans les quatre continents pour des rencontres scientifiques. J'ai eu aussi des séjours de post-doctorat dans des laboratoires européens et africains. La thèse m'a permis d'être accepté pour un contrat à durée déterminée de post-doctorat dans l'optique de continuer comme enseignant-chercheur au sein de la prestigieuse Université Polytechnique Mohammed VI de Benguérir, dans le département de la SAP+D (School of Architecture, Planning \& Design). Une université nouvelle et dynamique, un écosystème ouvert sur l'Afrique tout en imposant les critères et exigences de rang mondial. Le Maroc est aussi mon premier pays d'adoption. J'y ai vécu sept ans; je maîtrise très bien la langue locale, la culture et aussi en connais bien le climat. C'est d'après moi l'endroit idéal pour faire carrière; ce qui est pour moi l'occasion de rendre le peu que j'ai eu, les savoirs acquis. Je suis convaincu que c'est grâce à la thèse que cette opportunité m'a été offerte.

Par la suite j'ai été accepté en tant qu'enseignant-chercheur permanent dans l'une des rares écoles panafricaines inter-États du continent, l'École Africaine des Métiers de l'Architecture et de l'Urbanisme (EAMAU), sur la base d'un appel à candidature. Bientôt trois ans dans cette école et aujourd'hui co-coordonnateur d'un master 2 sur les transports et mobilité durable dans les villes africaines. Cette filière est ouverte à l'échelle du continent, en partenariat avec différents acteurs tels que le conservatoire national des arts et métiers de Paris (France), l'Université de Senghor d'Alexandrie (Égypte), la Coopération pour le Développement et l'Amélioration des Transports Urbains et Périurbains de France et le Centre d'Excellence sur les Villes Durables en Afrique de l'Université de Lomé (Togo). Enfin, depuis mai 2021, j'ai été nommé chef de division recherche de l'école africaine des métiers de l'architecture et de l'urbanisme. Je suis en charge du master recherche de l'école et je m'occupe de tout ce qui est planification, gestion et suivi de la recherche sous la supervision directe du directeur du développement et de la recherche au sein de l'EAMAU. Personne ne sait ce que l'avenir me réserve, mais j'espère toujours pouvoir être utile à l'Afrique qui a besoin de tous ses enfants dans tous les domaines.

21 Personne ne peut prédire l'avenir mais tout compte fait, on peut être prêt pour affronter le destin, comme beaucoup reste à faire sur le continent africain et dans tous les secteurs. L'essentiel, c'est de savoir bien nourrir sa thèse ; c'est-à-dire écrire, vivre sa passion, organiser et participer aux manifestations scientifiques d'abord par amour du métier, et puis aussi pour évoluer dans la carrière. Même si pour certains, le préfixe « Dr » sied à merveille et se suffit à lui-même, je rendrai ici un hommage à tous ceux qui ont d'une manière ou d'une autre participé à ma formation, à ce que je suis, depuis mon enfance de Sahélien cultivateur et berger devenu docteur et enseignant-chercheur.

Faites ce que vous voulez en toute honnêteté, aimez votre travail et vivez le présent. Vous gagnerez la confiance de ceux avec qui vous travaillerez, vos collègues, vos supérieurs et vos étudiants. À tout jeune en général et à un subsaharien en particulier : faites ce que vous pouvez lorsque vous vous engagez, vous apprendrez toujours quelque chose, quelles que soient les circonstances. Ne portez pas de jugements de valeur ni d'intolérance. Le travail paye, mais ce dernier doit être convaincant, irréfutable, même si parfois le résultat souhaité n'est pas au rendez-vous. Mais nous les Sahéliens en général et les Toubous $^{16}$ en particulier, nous sacrifions tout au monde, avec détermination, pour l'honneur et la dignité. Le travail joue toujours en votre faveur 
dans l'adaptation psychologique et/ou physique. Alors, c'est à vous de prendre le risque d'aller faire une thèse, de perdre quelques années, d'aller vous aventurer pour bâtir la bonne fondation d'une future carrière d'enseignant-chercheur.

\section{NOTES}

1. Howard S. Becker, Écrire les sciences sociales. Commencer et terminer son article, sa thèse ou son livre, Préface de Jean-Claude Passeron, Paris, Economica, 2004, 179 p.

2. Courbet Didier, Comment rédiger un projet de recherche (thèse de doctorat, mémoire de master...)? Institut de Recherche en Sciences de l'Information et de la Communication IRSIC/IMSIC, AixMarseille Université, juin 2017, version 3.

3. Zaki, Lamia, L'écriture d'une thèse en sciences sociales. Entre contingences et nécessités, Genèses 2006/4, nº 65, p. 112-125.

4. Emeline Eudes, «Trajectoire d'une écologie artistique: de l'inscription sur le paysage à l'effacement de la trace ", Marges [En ligne], 14 | 2012.

5. Natif de la ville de Faya-Largeau. La grande palmeraie du Nord du Tchad.

6. Le chadouf est un appareil à bascule servant à puiser l'eau d'un puits ou d'un cours d'eau. Il est souvent employé dans les zones agricoles irriguées dans les oasis et les palmeraies en zone sahélienne et saharienne.

7. Le Royaume Chérifien fait ici allusion à l'empire chérifien qui est une appellation historique du Maroc sous les régimes des dynasties des Saadiens et Alaouites, qui se revendiquent d'origine chérifienne.

8. Le salaire d'un ingénieur stagiaire en aménagement du territoire et urbanisme du MATUH dans la fonction publique du Tchad gagne à l'époque (2008) 145000 FCFA/mois. Ce qui fait sensiblement 223 euros le mois si l'on prend le taux d'échange à 650 FCFA pour 1 euro. Même en étant stagiaire au Maroc, je gagnais plus que cette somme.

9. Hemchi M. H., 2015, Mobilités urbaines et planification: le cas de N'Djamena, thèse de doctorat, Bordeaux, Université de Bordeaux-Montaigne, $475 \mathrm{p}$.

10. Les formations sont assurées par l'unité régionale de formation à l'information scientifique et technique (Urfist Bordeaux).

11. L'ensemble des années d'études supérieures entre le Maroc et la France.

12. https://tel.archives-ouvertes.fr/tel-01222332.

13. Howard S. Becker, Écrire les sciences sociales. Commencer et terminer son article, sa thèse ou son livre. Préface de Jean-Claude Passeron, Paris, Economica, 2004, 179 p.

14. Rivière Tiphaine, Carnets de thèse, Paris, Le Seuil, 2015, 180 p.

15. Les bureaux d'études, les collectivités locales, les associations locales, les organisations non gouvernementales, etc.

16. Les Toubous ou appelés aussi les Gouranes sont des peuples vivant dans le Sahara central aux frontières du Niger, du Tchad et de la Libye. Cette population vit du pastoralisme et du nomadisme. 


\section{AUTEUR}

\section{HASSANE MAHAMAT HEMCHI}

École Africaine des Métiers de l'Architecture et de l'Urbanisme - EAMAU 